\title{
Revisiting regularization with Kaluza-Klein states and Casimir vacuum energy from extra dimensional spaces
}

\author{
Hiroki Matsui ${ }^{1, *}$ and Yoshio Matsumoto ${ }^{2, \dagger}$ \\ ${ }^{1}$ Department of Physics, Tohoku University, Sendai, 980-8578 Japan \\ ${ }^{2}$ National Institute of Technology, Tsuyama College, Tsuyama, Okayama 708-0824, Japan
}

(Received 20 December 2018; published 18 July 2019)

\begin{abstract}
In the present paper, we investigate regularization of the one-loop quantum corrections with infinite Kaluza-Klein (KK) states and evaluate Casimir vacuum energy from extra dimensions. The extradimensional models always involve the infinite massless or massive Kaluza-Klein states, and therefore, the regularization of the infinite KK corrections is highly problematic. In order to avoid the ambiguity, we adopt the proper time integrals and the Riemann zeta function regularization in evaluating the summations of infinite KK states. In the calculation, we utilize the KK regularization method with exchanging the infinite summations and the infinite loop integrals. At the same time, we also evaluate the correction by the dimensional regularization method without exchanging the summations and the loop integrals. Then, we clearly show that the regularized Casimir corrections from the KK states have the form of $\propto 1 / R^{2}$ for the Higgs mass and $\propto 1 / R^{4}$ for the cosmological constant, where $R$ is the compactification radius. We also evaluate the Casimir energy in supersymmetric extradimensional models. The contributions from bulk fermions and bulk bosons are not offset because we choose supersymmetry breaking boundary conditions. The nonzero supersymmetric Casimir corrections from extra dimensions undoubtedly contribute to the Higgs mass and the cosmological constant. We conclude that the coefficients of such corrections are enhanced compared to the case without bulk supersymmetry.
\end{abstract}

DOI: $10.1103 /$ PhysRevD.100.016010

\section{INTRODUCTION}

The extra dimension has been discussed for more than 80 years. Famously, Kaluza and Klein [1,2] introduced an additional compactified dimension and suggested that our spacetime needs to have more than four dimensions to unify gravity and classical electrodynamics. Now, there have been many extradimensional models and theories to consider various problems of the particle physics. Especially, string theory [3] is a strong candidate to promise quantum gravity through seven additional spatial dimensions. In string theory (or brane-world scenario) we assume that the compactification scale of the extra dimensions is around $M_{\text {Planck }} \sim 10^{19} \mathrm{GeV}$. Naively, experimental observations of such extra dimensions are not realistic and we have no choice to extract constraints indirectly.

However, the hierarchy or naturalness problem [4-7] strongly circumscribes the possibility of the extradimensional

\footnotetext{
*hiroki.matsui.c6@tohoku.ac.jp

yoshio@cc.miyakonojo-nct.ac.jp

Present address: National Institute of Technology, Miyakonojo College, Miyakonojo, Miyazaki 885-0006, Japan.

Published by the American Physical Society under the terms of the Creative Commons Attribution 4.0 International license. Further distribution of this work must maintain attribution to the author(s) and the published article's title, journal citation, and DOI. Funded by SCOAP ${ }^{3}$.
}

models. As the most troublesome issues, there have existed the fine-tuning of the Higgs boson mass and the cosmological constant [8]. These dimensional parameters receive quantum corrections of the ultraviolet (UV) cutoff scale $\Lambda_{\mathrm{UV}}$ [9] and they quadratically or quartically diverge. ${ }^{1}$ These divergences should be regularized as the finite Casimir corrections depending on the first excited Kaluza-Klein (KK) scale $1 / R$ in the extradimensional models. The Higgs boson mass or the cosmological constant in the extra dimensional models is formally corrected from infinite KK states of bulk fields. The Kaluza-Klein regularization assumes exchanging loop integrals and summation of all the KK states, and then regularizes infinite divergences with KK summation using the zeta function formula. Adopting this method, we can regularize the infinite KK corrections and clearly obtain finite values that correspond to the Casimir energy from the extra dimension. However, the validity of the method is still under debate [13-23] and the cutoff sensitivity becomes more unclear.

The main purpose of this paper is to evaluate quantum radiative corrections for the Higgs mass with infinite $\mathrm{KK}$ states and clarify the Casimir vacuum energy from extra dimensions. In order to avoid the above ambiguity, in this paper, we evaluate one-loop quantum corrections from the

\footnotetext{
${ }^{1}$ Many new physics models have been considered [10-12] until now but there are no obvious solutions.
} 
extra dimensions with the KK regularization or the dimensional regularization. Then, we clearly show that the regularized finite corrections correspond to the Casimir energy from the extra dimensions. The regularized corrections from the KK states have the form of $\propto 1 / R^{2}$ for the Higgs mass and $\propto 1 / R^{4}$ for the cosmological constant (where $R$ is the compactification radius). The Casimir corrections can be considered as the quantum effects of compactified spaces, which is separated from the powerlaw divergences derived from the momentum cutoff or the pole for the dimensional regularization. Finally, we discuss supersymmetric or nonsupersymmetric Casimir vacuum energy from the extra dimensions.

The present paper is organized as follows. In Sec. II we introduce the Casimir energy from quantum zero-point energy with the Dirichlet boundary condition. We see that divergences from $d$-dimensional momentum integrals and infinite summations of discretized modes are regularized. In Sec. III we discuss the regularization issues for the Higgs mass and the cosmological constant in five-dimensional (5D) models with two boundaries and evaluate the radiative corrections from all the KK states of a bulk fermion. The divergences from the summation of KK states are regularized by the analytical continuation of the Riemann zeta function. However, we clearly show that the finite contributions correspond to the Casimir energy from extra dimensions. In Sec. IV we consider the case with supersymmetry (SUSY) and evaluate the Casimir quantum correction for the Higgs mass and the cosmological constant. We also discuss the Casimir corrections and divergent parts in suspersymmetric extra dimension. We comment on the difference of the treatment of divergent parts in the KK regularization and the dimensional regularization with SUSY. Finally, in Sec. V we draw the conclusion of this paper.

\section{CASIMIR ENERGY}

In this section we introduce the Casimir energy, which is formally defined as the quantum zero-point energy with the boundary conditions. The QFT [24] is formally constructed as an enormously large collection of the quantum harmonic oscillators. Thus, the vacuum energy with various quantum fields receives the divergent zero-point energy

$$
E_{\text {zero }}=\frac{ \pm 1}{2} \sum_{\text {spin }} \sum_{k} \omega_{k} \rightarrow \infty
$$

where $\omega_{k}=\sqrt{k^{2}+m^{2}}$ and $m$ is the masses of the boson and fermion fields. In principle, the divergent zero-point quantum corrections can be renormalized by the bare parameters and one fixes the finite physical parameters so that they agree with the observations, although the finetuning between the bare parameters and the quantum corrections would still be serious. In this sense, the QFT makes no prediction for the physical values of the vacuum energy or cosmological constant [25] and there exists no consensus about the reality of the zero-point energy in the community of the particle physics.

However, the difference of the divergent zero-point energy $\Delta E_{\text {zero }}$ has already been recognized to provide the observable effects. Famously, the Casimir effect [26] can be described by the zero-point electromagnetic energy between two parallel conducting plates and has been experimentally detected [27]. The difference of the divergent zero-point energy with the boundary conditions becomes finite and physical Casimir energy [28], and the phenomena have indeed provided an important hint of this problem.

Now, let us consider a massless scalar field between two parallel plates to impose the Dirichlet boundary condition,

$$
\phi(z=0)=\phi(z=a)=0 .
$$

This boundary condition discretizes the modes $k=n \pi / a$ and the zero-point energy on the condition can be given by

$$
E_{\text {zero }}=\frac{1}{2} \sum_{k} \omega_{k}=\frac{1}{2} \sum_{n=0}^{\infty} \int \frac{d^{2} k}{(2 \pi)^{2}} \sqrt{k^{2}+\left(\frac{n \pi}{a}\right)^{2}},
$$

which has some divergences.

Thus, let us adopt so-called zeta function regularization method using the following mathematical formula,

$\int_{0}^{\infty} \frac{d t}{t} t^{-\alpha} e^{-z t}=\Gamma(-\alpha) z^{\alpha}, \quad \int d^{d} k e^{-t k^{2}}=\left(\frac{\pi}{t}\right)^{d / 2}$,

where $d$ is the complex dimension of the spacetime and the left expression is called the proper time integral. Using these formulas, we can get the following expression,

$$
\begin{aligned}
E_{\text {zero }} & =\frac{1}{2} \sum_{n=0}^{\infty} \int \frac{d^{d} k}{(2 \pi)^{d}} \int_{0}^{\infty} \frac{d t}{t \cdot \Gamma(-1 / 2)} t^{-1 / 2} e^{-t\left(k^{2}+(n \pi / a)^{2}\right)} \\
& =-\frac{1}{4 \sqrt{\pi}} \frac{1}{(4 \pi)^{d / 2}} \sum_{n=0}^{\infty} \int_{0}^{\infty} \frac{d t}{t} t^{-1 / 2-d / 2} e^{-t n^{2} \pi^{2} / a^{2}} .
\end{aligned}
$$

Proceeding with the calculation we obtain

$$
\begin{aligned}
E_{\text {zero }} & =-\frac{1}{4 \sqrt{\pi}} \frac{1}{(4 \pi)^{d / 2}}\left(\frac{\pi}{a}\right)^{1+d} \Gamma\left(-\frac{d+1}{2}\right) \sum_{n=0}^{\infty} n^{d+1} \\
& =-\frac{1}{4 \sqrt{\pi}} \frac{1}{(4 \pi)^{d / 2}}\left(\frac{\pi}{a}\right)^{1+d} \Gamma\left(-\frac{d+1}{2}\right) \zeta(-d-1) .
\end{aligned}
$$

Now we take analytic continuation to remove the divergences and get the Riemann zeta function, 


$$
\Gamma\left(\frac{z}{2}\right) \zeta(z) \pi^{-z / 2}=\Gamma\left(\frac{1-z}{2}\right) \zeta(1-z) \pi^{-(1-z) / 2}
$$

The quantum zero-point energy on the Dirichlet boundary condition can be written as follows:

$$
\begin{aligned}
E_{\text {Casimir }} & =\lim _{d \rightarrow 2}\left\{-\frac{1}{2^{d+2} \pi^{d / 2+1}} \frac{1}{a^{d+1}} \Gamma\left(1+\frac{d}{2}\right) \zeta(2+d)\right\} \\
& =-\frac{\pi^{2}}{1440 \cdot a^{3}}
\end{aligned}
$$

where the zero-point divergences are removed by the analytic continuation of the zeta function $\zeta(z)$. The finite and negative contribution of the zero-point energy becomes so-called Casimir energy and observable as the attractive force between two parallel plates at small distances,

$$
F_{\text {Casimir }}=-\frac{\partial E_{\text {Casimir }}}{\partial a}=-\frac{\pi^{2}}{480 \cdot a^{4}},
$$

where $F_{\text {Casimir }}$ is a famous Casimir force per unit area. In the case of the electromagnetic fields between two parallel conductive plates, the Casimir force can be given as $F_{\text {Casimir }}=-\pi^{2} /\left(240 \cdot a^{4}\right)[26]$ where we count the two polarization states of the photon. The Casimir effect has been confirmed in many experiments [29-31] and strongly depends on the size, geometry, and topology of the given boundaries. On the other hand, whether the observations of the Casimir force prove the reality of the zero-point energy or not has still been under debate [32] because the Casimir force can alternatively be computed without invoking the zero-point electromagnetic energy as the standard perturbative methods of QED [32] like the Lamb shift and the van der Waals interactions [33-35]. The question of whether the zero-point energy exists or not is outside the scope of the present paper. However, the Casimir energy from geometrical conditions can be definitely defined and discussed as regards the corrections to the physical parameters. From here let us consider Casimir energy from extra dimensions and discuss its corrections for the Higgs mass and the cosmological constant.

\section{CASIMIR CORRECTIONS FROM EXTRA DIMENSIONS}

In this section, we discuss one-loop quantum corrections for the Higgs mass and the cosmological constant from the infinite KK states using the Kaluza-Klein regularization or the dimensional regularization.

\section{A. Casimir corrections for the Higgs mass with Kaluza-Klein regularization}

The KK regularization is one of the methods to regularize the divergences from infinite $\mathrm{KK}$ states and assumes the exchange between the loop integrals and the summation, and regularizes divergences of all the KK states using the zeta function formula.

Following the literature [13] let us discuss one-loop corrections for the Higgs mass in 5D models where the radius of the compactified space is $R$. The contributions from bulk fermions to the Higgs mass are expressed as the infinite summations of those of the $n$th KK states,

$$
Y^{2} \sum_{n=-\infty}^{\infty} \int_{0}^{\infty} \frac{d^{4} p}{(2 \pi)^{4}} \frac{1}{p^{2}+m_{n}^{2}}
$$

where $Y$ is the four-dimensional (4D) reduced Yukawa coupling of each fermionic KK state and assumed to be universal among different $n$th KK states. The $n$th KK mass eigenvalue is expressed as $m_{n}$ and we assume that the $n$th fermionic KK state has the KK mass eigenvalue $m_{n}=\frac{n}{R} \pi$.

Now, we utilize the following identities for the proper time integrals in the Schwinger representation as in the descriptions of [13],

$$
\int_{0}^{\infty} d t e^{-A t}=\frac{1}{A}, \quad \int d^{4} p e^{-p^{2} t}=\frac{\pi^{2}}{t^{2}} .
$$

Then, Eq. (10) is rewritten as

$$
\frac{Y^{2}}{16 \pi^{2}} \sum_{n=-\infty}^{\infty} \int_{0}^{\infty} \frac{d t}{t^{2}} e^{-\pi^{2} t n^{2} / R^{2}} .
$$

The above integrals have divergences at $t=0$. Now, we adopt the cutoff regularization method in calculating the loop integrals. We truncate $t$ by $1 / \Lambda^{2}$ at $t=0$. Additionally, we truncate $n$ by the cutoff KK number $l$ at $n=\infty$ (by $n=-l$ at $n=-\infty$ ). Here, we define the following integral $I_{l, \Lambda}$,

$$
I_{l, \Lambda}=\sum_{n=-l}^{l} \int_{1 / \Lambda^{2}}^{\infty} \frac{d t}{t^{2}} e^{-\pi^{2} t n^{2} / R^{2}} .
$$

Since the summation and the integral are finite in the above expression, we can exchange them safely,

$$
I_{l, \Lambda}=\int_{1 / \Lambda^{2}}^{\infty} d t \sum_{n=-l}^{l} \frac{1}{t^{2}} e^{-\pi^{2} t n^{2} / R^{2}} .
$$

Next, we can take the limit of $l \rightarrow \infty$ when $l \gg \Lambda R / \pi$ in Eq. (14),

$$
I_{\infty, \Lambda}=\int_{1 / \Lambda^{2}}^{\infty} d t \sum_{n=-\infty}^{\infty} \frac{1}{t^{2}} e^{-\pi^{2} t n^{2} / R^{2}}
$$

Now, we use the Poisson resummation formula, 


$$
\sum_{n=-\infty}^{\infty} e^{-\pi n^{2} x}=\frac{1}{\sqrt{x}} \sum_{w=-\infty}^{\infty} e^{-\frac{\pi w^{2}}{x}}
$$

where $w$ is the winding number of the bulk spacetime. Then, Eq. (15) becomes

$$
\begin{aligned}
I_{\infty, \Lambda} & =I_{w=\infty, \Lambda}=\frac{R}{\sqrt{\pi}} \int_{1 / \Lambda^{2}}^{\infty} d t t^{-\frac{5}{2}} \sum_{w=-\infty}^{\infty} e^{-\frac{R^{2}}{t} w^{2}} \\
& =\frac{R}{\sqrt{\pi}} \int_{1 / \Lambda^{2}}^{\infty} d t t^{-\frac{5}{2}}\left(1+2 \sum_{w=1}^{\infty} e^{-\frac{R^{2}}{t} w^{2}}\right) .
\end{aligned}
$$

Similarly to the process from Eq. (12) to Eq. (14), we again exchange the summation and the integral as

$I_{w=\infty, \Lambda}=\frac{R}{\sqrt{\pi}}\left(\int_{1 / \Lambda^{2}}^{\infty} d t t^{-\frac{5}{2}}+2 \sum_{w=1}^{\infty} \int_{1 / \Lambda^{2}}^{\infty} d t t^{-\frac{5}{2}} e^{-\frac{R^{2}}{t} w^{2}}\right)$.

The first term in the above equation is

$$
\frac{2 R}{3 \sqrt{\pi}} \Lambda^{3}
$$

When we take the limit $\Lambda \rightarrow \infty$, the first term diverges obviously. At the same time, the second term in Eq. (18) becomes

$$
\begin{aligned}
\frac{2 R}{\sqrt{\pi}} \sum_{w=1}^{\infty} \int_{0}^{\infty} d t t^{-\frac{5}{2}} e^{-\frac{R^{2}}{t} w^{2}} & =-\frac{2 R}{\sqrt{\pi}} \cdot \frac{1}{R^{3}} \sum_{w=1}^{\infty} \frac{1}{w^{3}} \cdot \int_{\infty}^{0} d y y^{\frac{1}{2}} e^{-y} \\
& =\frac{2}{\sqrt{\pi} R^{2}} \sum_{w=1}^{\infty} \frac{1}{w^{3}} \cdot \int_{0}^{\infty} d y y^{\frac{1}{2}} e^{-y} \\
& =\frac{2}{\sqrt{\pi} R^{2}} \zeta(3) \Gamma\left(\frac{3}{2}\right),
\end{aligned}
$$

where we transformed as $y=R^{2} w^{2} / t$. Eventually,

$$
\begin{aligned}
\frac{Y^{2}}{16 \pi^{2}} I_{\infty, \infty} & =\infty+\frac{Y^{2}}{8 \pi^{\frac{5}{2}} R^{2}} \zeta(3) \Gamma\left(\frac{3}{2}\right) \\
& =\infty+0.007612114264598318 Y^{2} / R^{2} .
\end{aligned}
$$

The second finite contribution corresponds to the Casimir energy for the one-loop corrections for the Higgs mass. At the finite cutoff scale $\Lambda$, the contributions from bulk fermions are expressed as

$$
\frac{Y^{2}}{16 \pi^{2}} I_{\infty, \Lambda}=\frac{Y^{2}}{24 \pi^{\frac{5}{2}}} R \Lambda^{3}+\frac{Y^{2}}{8 \pi^{\frac{5}{2}} R^{2}} \sum_{w=1}^{\infty} \frac{1}{w^{3}} \int_{0}^{R^{2} \Lambda^{2} w^{2}} d y y^{\frac{1}{2}} e^{-y} .
$$

We can easily check that the second term is finite at every $R>0$ or $\Lambda>0$.

\section{B. Casimir corrections for the Higgs mass with dimensional regularization}

However, there remain still some doubtful points in the method of the KK regularization [13-23]. The important point is the validity of exchanging the loop integrals and the KK summation because the infinite integrals and sums cannot always be exchanged. For instance, in the discussions of the literature [13], the regularized loop integrals $\int^{\Lambda} d p$ by the cutoff regularization and the KK summation $\Sigma_{n=-\infty(\text { or 0) }}^{\infty}$ truncated by the finite cutoff $l$ of the KK level $n$ are exchanged. Next, the authors take the limit of $\Lambda, l=\infty$ in the exchanged form of the corrections as follows:

$\sum_{n}^{\infty} \int_{0}^{\infty} d p \rightarrow \sum_{n}^{l} \int_{0}^{\Lambda} d p=\int_{0}^{\Lambda} d p \sum_{n}^{l} \rightarrow \int_{0}^{\infty} d p \sum_{n}^{\infty}$,

which seems to be correct. However, there is no proof of the KK regularization that the regularized values before exchanging and after exchanging are the same. Therefore, we must carefully check and confirm the validity of the KK regularization by calculating the KK corrections with another regularization method.

In order to avoid the above ambiguity of the KK regularization, we regularize the 4-momentum integrals by the method of dimensional regularization before summing all the KK states, following the paper [36]. Now, we define the proper time integral $I_{n}$ as follows:

$$
\begin{aligned}
I_{n} & \equiv \int \frac{d^{4} p}{(2 \pi)^{4}} \frac{1}{p^{2}+m_{n}^{2}} \\
& =\int \frac{d^{4} p}{(2 \pi)^{4}} \frac{1}{\Gamma(1)} \int_{0}^{\infty} d t t^{1-1} e^{-\left(p^{2}+m_{n}^{2}\right) t} \\
& =\int_{0}^{\infty} d t \frac{e^{-m_{n}^{2} t}}{(4 \pi t)^{4 / 2}} \\
& \sim \Gamma\left(1-\frac{d}{2}\right) \frac{\mu^{4-d}}{(4 \pi)^{d / 2}}\left(m_{n}^{2}\right)^{-\left(1-\frac{d}{2}\right)}(d \sim 4) \\
& \sim \Gamma\left(1-\frac{d}{2}\right) \frac{m_{n}^{2}}{(4 \pi)^{d / 2}}\left(\frac{m_{n}^{2}}{\mu^{2}}\right)^{\frac{d}{2}-2}, \quad(d \sim 4),
\end{aligned}
$$

where $\mu$ is the renormalization scale and $d$ is the complex dimension of the spacetime. We continue Eq. (24) analytically at $d=4$ and rewrite this expression with the infinitesimal parameter $\varepsilon=\frac{d}{2}-2$ as

$$
\begin{aligned}
I_{n} & =\Gamma(\varepsilon-1) \frac{m_{n}^{2}}{(4 \pi)^{\varepsilon+2}}\left(\frac{m_{n}^{2}}{\mu^{2}}\right)^{\varepsilon} \\
& =\left\{-\frac{1}{\varepsilon}+\gamma-1+(\cdots) \varepsilon+\mathcal{O}\left(\varepsilon^{2}\right)\right\}\left(\frac{m_{n}}{4 \pi}\right)^{2}\left(4 \pi \frac{\mu^{2}}{m_{n}^{2}}\right)^{\varepsilon},
\end{aligned}
$$


where $\gamma=0.577 \ldots$ is the Euler's constant. From

$$
\left(4 \pi \frac{\mu^{2}}{m_{n}^{2}}\right)^{\varepsilon} \sim 1+\varepsilon \ln \left(4 \pi \frac{\mu^{2}}{m_{n}^{2}}\right)+\mathcal{O}\left(\varepsilon^{2}\right)
$$

$I_{n}$ is rewritten as

$$
\begin{aligned}
I_{n}= & \left(\frac{m_{n}}{4 \pi}\right)^{2}\left\{-\frac{1}{\varepsilon}-1+\gamma-\ln \left(4 \pi \frac{\mu^{2}}{m_{n}^{2}}\right)\right. \\
& + \text { (negligible terms) }\} .
\end{aligned}
$$

Therefore, $I_{f}$ is given as follows:

$$
\begin{aligned}
I_{f} & =Y^{2} \sum_{n=-\infty}^{\infty} I_{n} \\
& =Y^{2} \sum_{n=-\infty}^{\infty}\left(\frac{m_{n}}{4 \pi}\right)^{2}\left\{-\frac{1}{\varepsilon}-1+\gamma+\ln \left(\frac{m_{n}^{2}}{4 \pi \mu^{2}}\right)\right\} .
\end{aligned}
$$

Now, we adopt the Riemann zeta function regularization to remove divergences of the quantum corrections,

$$
\begin{aligned}
\sum_{n=-\infty}^{\infty} n^{2} & =0+2 \sum_{n=1}^{\infty} n^{2}, \\
& =2 \zeta(-2) \\
& =0
\end{aligned}
$$

and

$$
\begin{aligned}
\sum_{n=-\infty}^{\infty} n^{2} \ln n^{2} & =0+2 \sum_{n=1}^{\infty} n^{2} \ln n^{2} \\
& =4 \sum_{n=1}^{\infty} n^{2} \ln n \\
& =-4 \zeta^{\prime}(-2),
\end{aligned}
$$

where $\zeta^{\prime}(x)$ is the differential of the zeta function $\zeta(x)$.

Therefore, the KK summed finite correction in Eq. (28) becomes

$$
\begin{aligned}
I_{\mathrm{f} \text { Casimir }} & =-\frac{Y^{2}}{4 R^{2}} \zeta^{\prime}(-2) \\
& =0.007612114264598319 \frac{Y^{2}}{R^{2}},
\end{aligned}
$$

which corresponds to the Casimir corrections for the Higgs mass from the KK states of bulk fermions. The value of Eq. (31) agrees highly accurately with the value of the KK regularization (21). On the other hand, the pole term in the expression of Eq. (28),

$$
-\frac{Y^{2}}{16 \pi^{2} R^{2}} \sum_{n=-\infty}^{\infty} n^{2} \cdot \frac{1}{\varepsilon}
$$

${ }^{2}$ must be treated carefully. If the limit $n \rightarrow \infty$ is faster than the limit $\varepsilon \rightarrow 0$, the term is exactly 0 by the relation of Eq. (29). However, when the limit $\varepsilon \rightarrow 0$ is faster than the limit $n \rightarrow \infty$, the term unavoidably diverges. In the latter case, this pole term must be renormalized by the bare parameter. Note that vanishing divergences in the above regularization have no bearing on the naturalness problem of the Higgs mass. Famously, the dimensional regularization reduces quadratic divergences, but the fine-tuning problem still exists and it is just appearance [9]. The infinite KK states exacerbate the problem rather than fourdimensional theory although the above regularization procedure hides the cutoff sensitivity.

\section{Casimir corrections for the cosmological constant with Kaluza-Klein regularization}

We consider the one-loop quantum corrections for the cosmological constant in 5D models with bulk fermions and discuss the Casimir energy from extra dimensions.

First, we evaluate with the Kaluza-Klein regularization. The zero-point vacuum energy derived from all the KK states of the bulk fermions is written as

$$
\rho_{\text {zero }}=\frac{1}{2} \sum_{n=-\infty}^{\infty} \int \frac{d^{3} k}{(2 \pi)^{3}} \sqrt{k^{2}+m_{n}^{2}}
$$

where $m_{n}=\pi \frac{n}{R}$ is the mass eigenvalue of the $n$th level KK state of the fermion. We use (4) again,

$$
\rho_{\text {zero }}=-\frac{1}{4 \sqrt{\pi}} \frac{1}{(4 \pi)^{3 / 2}} \sum_{n=-\infty}^{\infty} \int_{0}^{\infty} d t t^{-3} e^{-\frac{n^{2}}{R^{2}} \pi^{2} t} .
$$

Then, we permit the exchange of the infinite sum and the divergent integral,

$$
\rho_{\text {zero }}=-\frac{1}{32 \pi^{2}} \int_{0}^{\infty} d t t^{-3} \sum_{n=-\infty}^{\infty} e^{-\frac{n^{2}}{R^{2}} 2^{2} t}
$$

We use the Poissson resummation formula,

\footnotetext{
${ }^{2}$ In Ref. [37], a different analysis is made by the dimensional regularization. In this case, the UV divergences can be isolated instead of a separate treatment of the KK sum and 4D momentum integral. They can isolate divergences by exchanging sum and integral in 5D dimensional regularization (see Appendix A of [37]), and the divergences are canceled by higher dimensional operators.
} 


$$
\begin{aligned}
\rho_{\text {zero }} & =-\frac{1}{32 \pi^{2}} \int_{0}^{\infty} d t t^{-3} \cdot \frac{R}{\sqrt{\pi t}}\left(1+2 \sum_{w=1}^{\infty} e^{-\frac{R^{2}}{t} w^{2}}\right) \\
& =-\frac{R}{32 \pi^{5 / 2}} \int_{0}^{\infty} d t t^{-7 / 2}\left(1+2 \sum_{w=1}^{\infty} e^{-\frac{R^{2}}{t} w^{2}}\right) .
\end{aligned}
$$

Now, we truncate $t$ by the finite cutoff $t=1 / \Lambda^{2}$ at $t=0$.

$$
\begin{aligned}
\rho_{\text {zero }} & =-\frac{R}{32 \pi^{5 / 2}}\left(\int_{1 / \Lambda^{2}}^{\infty} d t t^{-7 / 2}+2 \sum_{w=1}^{\infty} \int_{1 / \Lambda^{2}}^{\infty} d t t^{-7 / 2} e^{-\frac{R^{2}}{t} w^{2}}\right) \\
& =-\frac{R \Lambda^{5}}{112 \pi^{5 / 2}}-\frac{R}{16 \pi^{5 / 2}} \sum_{w=1}^{\infty} \int_{1 / \Lambda^{2}}^{\infty} d t t^{-7 / 2} e^{-\frac{R^{2}}{t} w^{2}} .
\end{aligned}
$$

When we take the limit $\Lambda \rightarrow \infty$,

$$
\begin{aligned}
\rho_{\text {zero }} & =-\infty-\frac{1}{16 \pi^{5 / 2} R^{4}} \sum_{w=1}^{\infty} \frac{1}{w^{5}} \int_{0}^{\infty} d y y^{3 / 2} e^{-y} \\
& =-\infty-\frac{1}{16 \pi^{5 / 2} R^{4}} \zeta(5) \Gamma\left(\frac{5}{2}\right) .
\end{aligned}
$$

The second finite term is the Casimir correction for the vacuum energy and written as

$$
\therefore \rho_{\text {Casimir }}=-0.0049248162891899275 / R^{4} \text {. }
$$

\section{Casimir corrections for the cosmological constant with dimensional regularization}

Second, we evaluate the one-loop quantum corrections for the cosmological constant in 5D models with the dimensional regularization.

Let us consider the case of the fourth dimension for simplicity. Adopting the dimensional regularization, the quantum corrections of the cosmological constant from the zero-point energy are given by

$$
\begin{aligned}
\rho_{\text {zero }} & =\frac{E_{\text {zero }}}{\text { Volume }}=\frac{1}{2} \int \frac{d^{3} k}{(2 \pi)^{3}} \sqrt{k^{2}+m^{2}} \\
& =\frac{m^{4}}{64 \pi^{2}}\left\{\ln \left(\frac{m^{2}}{\mu^{2}}\right)-\frac{1}{\varepsilon}-\log 4 \pi+\gamma-\frac{3}{2}\right\} .
\end{aligned}
$$

Then, we divide the bare cosmological constant vacuum term $\rho_{\Lambda}$ to be $\rho_{\Lambda}=\rho_{\Lambda}(\mu)+\delta \rho_{\Lambda}$, where $\rho_{\Lambda}=\Lambda / 8 \pi G_{N}$ is defined by the cosmological constant $\Lambda$ and the Newton's constant $G_{N}$. The counterterm $\delta \rho_{\Lambda}$ is written as

$$
\delta \rho_{\Lambda}=\frac{m^{4}}{4(4 \pi)^{2}}\left(\frac{1}{\varepsilon}+\log 4 \pi-\gamma\right),
$$

where we adopt the $\overline{\mathrm{MS}}$ scheme. Absorbing divergences into the counterterm $\delta \rho_{\Lambda}$, we obtain the following renormalized expression,

$$
\begin{aligned}
\rho_{\text {vacuum }}= & \rho_{\Lambda}(\mu)+\delta \rho_{\Lambda} \\
& +\frac{m^{4}}{64 \pi^{2}}\left\{\ln \left(\frac{m^{2}}{\mu^{2}}\right)-\frac{1}{\varepsilon}-\log 4 \pi+\gamma-\frac{3}{2}\right\} \\
= & \rho_{\Lambda}(\mu)+\frac{m^{4}}{64 \pi^{2}}\left(\ln \frac{m^{2}}{\mu^{2}}-\frac{3}{2}\right),
\end{aligned}
$$

where the divergences of the zero-point vacuum energy are definitely renormalized by the cosmological constant term. In the standard model (SM) framework, the vacuum energy density can be written as follows:

$$
\begin{aligned}
\rho_{\text {vacuum }}= & \rho_{\Lambda}(\mu)+\rho_{\mathrm{EW}}+\rho_{\mathrm{QCD}} \\
& +\sum_{i} \frac{n_{i} m_{i}^{4}}{64 \pi^{2}}\left(\ln \frac{m_{i}^{4}}{\mu^{2}}-\frac{3}{2}\right)+\mathcal{O}\left(\Lambda_{\mathrm{UV}}^{4}\right)+\cdots,
\end{aligned}
$$

where $\rho_{\mathrm{EW}}$ or $\rho_{\mathrm{QCD}}$ express the classical vacuum energies of the electroweak or chiral symmetry breaking. $n_{i}$ and $m_{i}$ are the number of degrees of freedom and the mass of the SM particle $i$, respectively. However, the dark energy [38-40] as the current physical value of the vacuum energy is extremely small. The fine-tuning of the vacuum energy is highly problematic and we have no satisfactory solutions to derive such an extremely small scale.

Next, we evaluate the quantum corrections for the cosmological constant in 5D models with bulk fermions. The zero-point vacuum energy derived from all the KK states of the bulk fermions is written as

$$
\rho_{\text {zero }}=\frac{1}{2} \sum_{n=-\infty}^{\infty} \int \frac{d^{3} k}{(2 \pi)^{3}} \sqrt{k^{2}+m_{n}^{2}}
$$

where $m_{n}=\pi \frac{n}{R}$ is the mass eigenvalue of the $n$th level KK state of the fermion. Then, we rewrite the renormalized expression of Eq. (42) as

$$
\begin{aligned}
\rho_{\text {vacuum }} & =\rho_{\Lambda}(\mu)+\sum_{n=-\infty}^{\infty} \frac{m_{n}{ }^{4}}{64 \pi^{2}}\left(\ln \frac{m_{n}{ }^{2}}{\mu^{2}}-\frac{3}{2}\right) \\
& =\rho_{\Lambda}(\mu)+\sum_{n=-\infty}^{\infty} \frac{\pi^{2} n^{4}}{64 R^{4}}\left\{\ln n^{2}+\ln \left(\frac{\pi^{2}}{\mu^{2} R^{2}}\right)-\frac{3}{2}\right\} .
\end{aligned}
$$

The KK summed term can be evaluated by the Riemann zeta function regularization,

$$
\sum_{n=-\infty}^{\infty} n^{4}=0+2 \sum_{n=1}^{\infty} \frac{1}{n^{-4}}=2 \zeta(-4)=0
$$

and 


$$
\begin{aligned}
\sum_{n=-\infty}^{\infty} n^{4} \ln n^{2} & =0+2 \sum_{n=1}^{\infty} n^{4} \ln n^{2} \\
& =4 \sum_{n=1}^{\infty} \frac{1}{n^{-4}} \ln n \\
& =-4 \zeta^{\prime}(-4)
\end{aligned}
$$

where $\zeta^{\prime}(x)$ is the differential of the zeta function $\zeta(x)$ $\left(\zeta^{\prime}(-4)=0.00798381\right)$. Thus, the KK summed finite contribution in Eq. (45) becomes

$$
\begin{aligned}
\rho_{\text {Casimir }} & =\frac{\pi^{2}}{64 R^{4}} \cdot\left\{-4 \zeta^{\prime}(-4)\right\} \\
& =-0.004924816289189928 / R^{2},
\end{aligned}
$$

which corresponds to the physical Casimir correction from the extra dimension. The result agrees very precisely (but not completely equally) with the KK regularization on the value of the correction (39).

\section{CASIMIR CORRECTIONS FROM SUPERSYMMETRIC EXTRA DIMENSIONS}

Famously, SUSY can in principle remove quadratic or quartic divergences of the quantum radiative corrections. However, our real world must break the SUSY and the Higgs mass receives quadratic divergent corrections up to the breaking scale. The zero-point energy completely cancels out in the SUSY case due to its opposite signs of the boson and fermion. However, supersymmetric Casimir energy is still nonzero [37,41-43] because the boundary conditions break the SUSY. As previously discussed in Sec. III, the quantum corrections from extra dimensions correspond to the Casimir energy, and therefore, the SUSY cannot reduce the Casimir quantum corrections from the infinite $\mathrm{KK}$ states of bulk fields.

\section{A. Supersymmetric Casimir corrections for the Higgs mass with Kaluza-Klein regularization}

Next, we consider bulk supersymmetry and discuss bosonic contributions additionally. The bosonic contributions are written as [13]

$$
g^{2} \int \frac{d^{4} p}{(2 \pi)^{4}} \frac{1}{p^{2}+m_{n}^{\prime 2}},
$$

where $g$ is the 4D reduced coupling of the bulk SUSY multiplet and the Higgs boson, and it is assumed to be universal among different KK bosonic and fermionic states. The bosonic KK mass eigenvalues are expressed as $m_{n}^{\prime}$. Now, we impose the boundary conditions for the bosonic KK modes to have the SUSY breaking KK mass eigenvalues $m_{n}^{\prime}=\frac{\pi}{R}\left(n+\frac{1}{2}\right)$. Similarly to Eq. (12),

$$
\begin{aligned}
g^{2} & \sum_{n=-\infty}^{\infty} \int_{0}^{\infty} \frac{d p^{4}}{\left(2 \pi^{4}\right)} \frac{1}{p^{2}+m_{n}^{\prime 2}} \\
& =\frac{g^{2}}{16 \pi^{2}} \sum_{n=-\infty}^{\infty} \int_{0}^{\infty} \frac{d t}{t^{2}} e^{-\pi^{2} t\left(n+\frac{1}{2}\right)^{2} / R^{2}}
\end{aligned}
$$

We define the following integral

$$
I_{l, \Lambda}^{b}=\sum_{n=-l}^{l} \int_{1 / \Lambda^{2}}^{\infty} \frac{d t}{t^{2}} e^{-\pi^{2} t\left(n+\frac{1}{2}\right)^{2} / R^{2}} .
$$

When we take the limit $l \rightarrow \infty$,

$$
I_{\infty, \Lambda}^{b}=\int_{1 / \Lambda^{2}}^{\infty} d t \sum_{n=-\infty}^{\infty} \frac{1}{t^{2}} e^{-\pi^{2} t\left(n+\frac{1}{2}\right)^{2} / R^{2}}
$$

The Poisson resummation formula is rewritten as

$$
\begin{aligned}
\sum_{n=-\infty}^{\infty} e^{-\pi\left(n+\frac{1}{2}\right)^{2} x} & =\frac{1}{\sqrt{x}} \sum_{w=-\infty}^{\infty} e^{\pi i w-\pi \frac{w^{2}}{x}} \\
& =\frac{1}{\sqrt{x}} \sum_{w=-\infty}^{\infty}(-1)^{w} e^{-\pi \frac{w^{2}}{x}}
\end{aligned}
$$

Then, Eq. (52) becomes

$$
\begin{aligned}
I_{\infty, \Lambda}^{b} & =I_{w=\infty, \Lambda}^{b} \\
& =\frac{R}{\sqrt{\pi}} \int_{1 / \Lambda^{2}}^{\infty} d t t^{-\frac{5}{2}} \sum_{w=-\infty}^{\infty}(-1)^{w} e^{-\frac{R^{2}}{t} w^{2}} \\
& =\frac{R}{\sqrt{\pi}} \int_{1 / \Lambda^{2}}^{\infty} d t t^{-\frac{5}{2}}\left(1+2 \sum_{w=1}^{\infty}(-1)^{w} e^{-\frac{R^{2}}{t} w^{2}}\right) .
\end{aligned}
$$

When we take the limit $\Lambda \rightarrow \infty$ and the fermionic contributions are written as

$$
g^{2} \sum_{n=-\infty}^{\infty} \int_{0}^{\infty} \frac{d p^{4}}{(2 \pi)^{4}} \frac{1}{p^{2}+\left(\frac{n}{R} \pi\right)^{2}}
$$

the finite Casimir correction parts in the contributions from both fermionic and bosonic modes are summed up as

$$
\begin{aligned}
& \frac{g^{2}}{16 \pi^{2}} \cdot \frac{2}{\sqrt{\pi} R^{2}} \sum_{w=1}^{\infty} \frac{1-(-1)^{w}}{w^{3}} \cdot \int_{0}^{\infty} d y y^{\frac{1}{2}} e^{-y} \\
& \quad=\frac{g^{2}}{8 \pi^{\frac{5}{2}} R^{2}} \cdot \frac{7}{4} \zeta(3) \Gamma\left(\frac{3}{2}\right) \\
& =0.013321199963047056 g^{2} / R^{2} .
\end{aligned}
$$

The power-law contributions $\left(\propto R \Lambda^{3}\right)$ are exactly offset by the bulk supersymmetry, which is broken softly by the boundary conditions. Because of the boundary conditions, the Casimir energy appears and its coefficient is enhanced 
compared to the case with only fermionic modes. As later discussed in Sec. IV, this fact that the Casimir energy is enhanced with the SUSY is seen in the case of the dimensional regularization.

\section{B. Supersymmetric Casimir corrections for the Higgs mass with the dimensional regularization}

In this section, we evaluate one-loop corrections for the Higgs mass from fermionic and bosonic KK modes in 5D supersymmetric models and clearly discuss the supersymmetric Casimir corrections from the extra dimensions. We choose boundary conditions that bulk bosons have SUSY breaking KK mass eigenvalues, and one-loop contributions from all the KK states of the bulk boson are written as

$$
g^{2} \int \frac{d^{4} p}{(2 \pi)^{4}} \frac{1}{p^{2}+m_{n}^{\prime 2}},
$$

where $m_{n}^{\prime}=\frac{\pi}{R}\left(n+\frac{1}{2}\right)$. This is calculated by substituting $n+\frac{1}{2}$ for $n$ in $I_{f}$. To do so, we must evaluate the following quantities:

$$
\sum_{n=-\infty}^{\infty}\left(n+\frac{1}{2}\right)^{2} \& \sum_{n=-\infty}^{\infty}\left(n+\frac{1}{2}\right)^{2} \ln \left(n+\frac{1}{2}\right)^{2}
$$

The first quantity can be exprressed as 0 by Hurvitz zeta function $\zeta\left(-2, \frac{1}{2}\right)=0$. The second quantity is

$$
\sum_{n=-\infty}^{-1}\left(n+\frac{1}{2}\right)^{2} \ln \left(n+\frac{1}{2}\right)^{2}+\sum_{n=0}^{\infty}\left(n+\frac{1}{2}\right)^{2} \ln \left(n+\frac{1}{2}\right)^{2}
$$

The first term in this expression is

$\sum_{n=1}^{\infty}\left(n-\frac{1}{2}\right)^{2} \ln \left(n-\frac{1}{2}\right)^{2}=\frac{1}{4} \ln \frac{1}{4}+\frac{9}{4} \ln \frac{9}{4}+\cdots$.

This is equal to the second term that is $2 \zeta^{\prime}\left(-2, \frac{1}{2}\right)$ (the differential of Hurvitz zeta function). Therefore, the second quantity in Eq. (58) is $4 \zeta^{\prime}\left(-2, \frac{1}{2}\right)$. So the KK summed finite parts in the bosonic contributions are

$$
\left.I_{b}\right|_{\mathrm{KK}}=\frac{g^{2}}{(4 R)^{2}} \cdot 4 \zeta^{\prime}\left(-2, \frac{1}{2}\right)=\frac{g^{2}}{4 R^{2}} \zeta^{\prime}\left(-2, \frac{1}{2}\right)
$$

As we see, the finite Casimir correction parts derived from the contributions of all the KK states are written as

$$
\begin{aligned}
I_{\mathrm{f}}-I_{\mathrm{b}} & =\left.I_{f}\right|_{\mathrm{KK}}-\left.I_{b}\right|_{\mathrm{KK}} \\
& =-\frac{g^{2}}{4 R^{2}}\left\{\zeta^{\prime}(-2)-\zeta^{\prime}\left(-2, \frac{1}{2}\right)\right\} \\
& =0.013321199963047058 g^{2} / R^{2} .
\end{aligned}
$$

The result agrees highly accurately with the value of the KK regularization (56).

\section{Supersymmetric Casimir corrections for the cosmological constant with the Kaluza-Klein regularization}

Similarly, the Casimir vacuum energy in 5D supersymmetric models can be evaluated. First, we calculate with the Kaluza-Klein regularization. We assume the KK mass eigenvalues are the same as Sec. IVA. The Casimir vacuum energy is evaluated as the difference of fermionic and bosonic contributions,

$$
\begin{aligned}
\rho_{\text {Casimir }} & =-\frac{1}{16 \pi^{5 / 2} R^{4}} \sum_{w=1}^{\infty} \frac{1-(-1)^{w}}{w^{5}} \int_{0}^{\infty} d y y^{3 / 2} e^{-y} \\
& =-\frac{1}{16 \pi^{5 / 2} R^{4}} \cdot \frac{31}{16} \zeta(5) \Gamma\left(\frac{5}{2}\right) \\
& =-0.009541831560305483 / R^{4} .
\end{aligned}
$$

We can see that the coefficient of the Casimir vacuum energy is enhanced the same as is the case with the Higgs mass.

\section{Supersymmetric Casimir corrections for the cosmological constant with the dimensional regularization}

Next, we evaluate the Casimir vacuum energy with the dimensional regularization. Fermionic and bosonic contributions are calculated by Eq. (45). The KK summed corrections are regularized by the zeta function regularization. Eventually, the Casimir vacuum energy is evaluated as

$$
\begin{aligned}
\rho_{\text {Casimir }} & =\frac{\pi^{2}}{64 R^{4}} \cdot\left[-4\left\{\zeta^{\prime}(-4)-\zeta^{\prime}\left(-4, \frac{1}{2}\right)\right\}\right] \\
& =-0.009541831560305485 / R^{4} .
\end{aligned}
$$

The result agrees highly accurately with the value of the KK regularization (63).

In both the case of the Higgs mass and the vacuum energy, the infinite summations of the products of $m_{n}^{2}$ or $m_{n}^{4}$ and the poles $1 / \varepsilon$ diverge when we regularize by the method of the dimensional regularization if the limit $\varepsilon \rightarrow 0$ is earlier than the limit of $n \rightarrow \infty$ even with supersymmetry. In the cutoff regularization, the corrections for the Casimir vacuum energy with SUSY are written as 


$$
\begin{aligned}
\rho_{\text {zero }}= & \frac{1}{16 R^{2}} \sum_{n=-\infty}^{\infty}\left\{n^{2}-\left(n+\frac{1}{2}\right)^{2}\right\} \Lambda_{\mathrm{UV}}^{2} \\
& +\frac{\pi^{2}}{64 R^{4}} \sum_{n=-\infty}^{\infty}\left\{n^{4} \ln \left(\frac{n^{2}}{\Lambda_{\mathrm{UV}}^{2}}\right)-\left(n+\frac{1}{2}\right)^{4} \ln \left(\frac{n+\frac{1}{2}}{\Lambda_{\mathrm{UV}}}\right)^{2}\right\} \\
& +\cdots
\end{aligned}
$$

In this expression, the contributions of $\Lambda^{4}$ do not appear because SUSY is broken softly by the boundary conditions.

\section{SUMMARY AND CONCLUSION}

In the present paper, we have reinvestigated regularization of the one-loop quantum corrections for the Higgs mass and the cosmological constant in 5D spacetime with infinite $\mathrm{KK}$ states and discussed the Casimir corrections from extra dimensions. We evaluated the corrections by the $\mathrm{KK}$ regularization and the dimensional regularization. We found that the Casimir corrections of the KK regularization and the dimensional regularization match with very high accuracy, but are not completely the same values. These subtle differences may be caused by whether the infinite momentum integrals and the infinite KK summations are exchanged or not.

We have also evaluated the Casimir corrections in supersymmetric extradimensional models. The contributions from bulk fermions and bulk bosons (components of bulk SUSY multiplets) are not offset because the general boundary conditions break SUSY. The supersymmetric Casimir corrections from extra dimensions are still nonzero and undoubtedly contribute to the Higgs mass and the cosmological constant.

We have also got the result that the coefficients of the finite Casimir corrections are enhanced a little with bulk supersymmetry (broken by boundary conditions) compared to the case with only fermionic modes in the calculations of both the Higgs boson and the vacuum energy. This fact is seen when we calculate both with the KK regularization and the dimensional regularization method.

Furthermore, we have also discussed the regularization issues of the extra dimension. In the dimensional regularization, the infinite KK summations of the products of $n^{2}$ or $n^{4}$ and poles $1 / \varepsilon$ have divergences that must be renormalized away by the counterterms unlike in the case of the cutoff regularization where the power-law contributions of cutoff $\Lambda$ are offset exactly even with SUSY breaking boundary conditions.

\section{ACKNOWLEDGMENTS}

We thank Yoshiyuki Tatsuta for helpful comments and discussions.
[1] T. Kaluza, Sitzungsber. Preuss. Akad. Wiss. Berlin (Math. Phys.) 1921, 966 (1921) [Int. J. Mod. Phys. D 27, 1870001 (2018)].

[2] O. Klein, Z. Phys. 37, 895 (1926).

[3] P. Horava and E. Witten, Nucl. Phys. B460, 506 (1996).

[4] K. G. Wilson, Phys. Rev. D 3, 1818 (1971).

[5] E. Gildener, Phys. Rev. D 14, 1667 (1976).

[6] S. Weinberg, Phys. Lett. 82B, 387 (1979).

[7] G. 't Hooft, NATO Sci. Ser. B 59, 135 (1980).

[8] S. Weinberg, Rev. Mod. Phys. 61, 1 (1989).

[9] G. F. Giudice, Proc. Sci., EPS-HEP2013 (2013) 163.

[10] L. Randall and R. Sundrum, Phys. Rev. Lett. 83, 3370 (1999).

[11] P. W. Graham, D. E. Kaplan, and S. Rajendran, Phys. Rev. Lett. 115, 221801 (2015).

[12] H. Matsui and Y. Matsumoto, arXiv:1608.08838.

[13] T. Kobayashi and H. Terao, Prog. Theor. Phys. 107, 785 (2002).

[14] R. Contino and L. Pilo, Phys. Lett. B 523, 347 (2001).

[15] D. M. Ghilencea and H.-P. Nilles, Phys. Lett. B 507, 327 (2001).

[16] J. Kubo, H. Terao, and G. Zoupanos, Nucl. Phys. B574, 495 (2000).

[17] D. M. Ghilencea, H. P. Nilles, and S. Stieberger, New J. Phys. 4, 15 (2002).
[18] H. D. Kim, arXiv:hep-ph/0106072.

[19] I. Antoniadis, K. Benakli, and M. Quiros, New J. Phys. 3, 20 (2001).

[20] A. Delgado, G. von Gersdorff, and M. Quiros, Nucl. Phys. B613, 49 (2001).

[21] R. Barbieri, L. J. Hall, and Y. Nomura, Phys. Rev. D 63, 105007 (2001).

[22] I. Antoniadis, J. R. Ellis, A. B. Lahanas, and D. V. Nanopoulos, Phys. Lett. B 241, 24 (1990).

[23] I. L. Buchbinder, S. D. Odintsov, and O. A. Fonarev, Pis'ma Zh. Eksp. Teor. Fiz. 51, 343 (1990) [Phys. Lett. B 245, 365 (1990)].

[24] M. Born, W. Heisenberg, and P. Jordan, Z. Phys. 35, 557 (1926).

[25] M. Maggiore, Phys. Rev. D 83, 063514 (2011).

[26] H. B. G. Casimir, Kon. Ned. Akad. Wetensch. Proc. 100N34, 61 (1997) [Indagat. Math 10, 261 (1948)].

[27] S. K. Lamoreaux, Phys. Rev. Lett. 78, 5 (1997); 81, 5475(E) (1998).

[28] M. Bordag, U. Mohideen, and V. M. Mostepanenko, Phys. Rep. 353, 1 (2001).

[29] U. Mohideen and A. Roy, Phys. Rev. Lett. 81, 4549 (1998).

[30] A. Roy, C.-Y. Lin, and U. Mohideen, Phys. Rev. D 60, 111101 (1999). 
[31] G. Bressi, G. Carugno, R. Onofrio, and G. Ruoso, Phys. Rev. Lett. 88, 041804 (2002).

[32] R. L. Jaffe, Phys. Rev. D 72, 021301 (2005).

[33] E. M. Lifshitz, Sov. Phys. JETP 2, 73 (1956).

[34] I. E. Dzyaloshinskii, E. M. Lifshitz, and L. P. Pitaevskii, Sov. Phys. Usp. 4, 153 (1961).

[35] J. S. Schwinger, L. L. DeRaad, Jr., and K. A. Milton, Ann. Phys. (N.Y.) 115, 1 (1978).

[36] A. Gupta, arXiv:hep-th/0210069.

[37] D. M. Ghilencea and H. M. Lee, J. High Energy Phys. 09 (2005) 024.
[38] S. Perlmutter et al. (Supernova Cosmology Project), Nature (London) 391, 51 (1998).

[39] A. G. Riess et al. (Supernova Search Team), Astron. J. 116, 1009 (1998).

[40] N. A. Bahcall, J. P. Ostriker, S. Perlmutter, and P. J. Steinhardt, Science 284, 1481 (1999).

[41] H. Abe, J. Hashida, T. Muta, and A. Purwanto, Mod. Phys. Lett. A 14, 1033 (1999).

[42] D. M. Ghilencea, J. High Energy Phys. 03 (2005) 009.

[43] D. M. Ghilencea, D. Hoover, C. P. Burgess, and F. Quevedo, J. High Energy Phys. 09 (2005) 050. 